\title{
accretion flow and Disparate Profiles of Raman Scattered O Vi $\lambda \lambda$ 1032, 1038 In The SYMbiotic STAR V1016 Cygni
}

\author{
Jeong-Eun Heo And Hee-Won Lee \\ Department of Physics and Astronomy, Sejong University, 209 Neungdong-ro, Gwangjin-gu, Seoul 143-747, Korea \\ jeung6145@gmail.com, hwlee@sejong.ac.kr
}

Received January 20, 2015; accepted February 26, 2015

\begin{abstract}
The symbiotic star V1016 Cygni, a detached binary system consisting of a hot white dwarf and a mass-losing Mira variable, shows very broad emission features at around $6825 \AA$ and $7082 \AA$, which are Raman scattered O VI $\lambda \lambda$ 1032, 1038 by atomic hydrogen. In the high resolution spectrum of V1016 Cyg obtained with the Bohyunsan Optical Echelle Spectrograph these broad features exhibit double peak profiles with the red peak stronger than the blue counterpart. However, their profiles differ in such a way that the blue peak of the 7082 feature is relatively weaker than the 6825 counterpart when the two Raman features are normalized to exhibit an equal red peak strength in the Doppler factor space. Assuming that an accretion flow around the white dwarf is responsible for the double peak profiles, we attribute this disparity in the profiles to the local variation of the flux ratio of O VI $\lambda \lambda 1032,1038$ in the accretion flow. A Monte Carlo technique is adopted to provide emissivity maps showing the local emissivity of O VI $\lambda 1032$ and $\mathrm{O}$ VI $\lambda 1038$ in the vicinity of the white dwarf. We also present a map indicating the differing flux ratios of O VI $\lambda \lambda 1032$ and 1038. Our result shows that the flux ratio reaches its maximum of 2 in the emission region responsible for the central trough of the Raman feature and that the flux ratio in the inner red emission region is almost 1 . The blue emission region and the outer red emission region exhibit an intermediate ratio around 1.5. We conclude that the disparity in the profiles of the two Raman O VI features strongly implies accretion flow around the white dwarf, which is azimuthally asymmetric.
\end{abstract}

Key words: binaries: symbiotic — stars: individual: V1016 Cyg — scattering — accretion, accretion disks - line: profiles

\section{INTRODUCTION}

Active binary systems containing a white dwarf are important as potential progenitors of Type Ia supernovae, which were essential in establishing the standard picture of the universe dominated by dark energy (e.g., Iben \& Tutukov 1984; Perlmutter et al. 1998). Depending on the nature of the companion, active white dwarf binary systems can be divided into cataclysmic variables and symbiotic stars. Cataclysmic variables are binary systems of a white dwarf with a late type main sequence star that fills the Roche lobe. Prominent activities exhibited by cataclysmic variables are attributed to the presence of an accretion disk around the white dwarf that results from the Roche lobe overflow from the companion (e.g., Warner 1995).

Symbiotic stars are binary systems of a white dwarf and a mass losing giant which does not fill the Roche lobe (Mikolajewska 2012). The spectra of symbiotic stars are characterized by the existence of TiO absorption bands indicative of a cool star and prominent emission lines including high ionization species such as $\mathrm{O}$ VI and Ne VII. Considering the size of the giant companion, the orbital period usually exceeds several hundred days and sometimes several decades. They are classified into ' $S$ ' type and ' $D$ ' type, where ' $D$ ' type symbiotics exhibit an IR excess that indicates the presence of a

CORRESPONDING AUTHOR: H.-W. Lee warm dust component with a temperature $T \sim 1000 \mathrm{~K}$ (Angeloni et al. 2010). In contrast, the IR excess is absent in the spectral energy distribution of ' $\mathrm{S}$ ' type symbiotics.

One distinguishing aspect of the symbiotic spectra is the existence of broad emission features at around $6825 \AA$ and $7082 \AA$, whose existence is quite exclusive to symbiotic stars with an exception of a young planetary nebula (e.g., Allen 1980). These features were identified by Schmid (1989), who proposed that they are formed through Raman scattering of O VI $\lambda \lambda 1032$, 1038 by atomic hydrogen. When an O vi $\lambda 1032$ line photon is incident upon a hydrogen atom in the ground state, an optical photon at around $6825 \AA$ is re-emitted as the hydrogen atom de-excites to the excited $2 s$ state. In a similar way, an optical photon at around $7082 \AA$ results from the inelastic scattering process for an O vi $\lambda$ 1038 photon. The cross sections and branching ratios for Raman scattering can be computed in a straightforward manner using the Kramers-Heisenberg formula (e.g., Schmid 1989; Bach \& Lee 2014).

Raman $\mathrm{O}$ vi features in symbiotic stars are known to exhibit complicated profiles including double-peak profiles and triple-peak profiles (e.g., Harries \& Howarth 1997). In particular, a number of symbiotic stars classified as symbiotic novae including V1016 Cyg, HM Sge and RR Tel exhibit double-peak profiles clearly with the red peak stronger than the blue counterpart. 
One possible explanation for the double peaked structure is that the scattering occurs in two distinct regions one of which approaches the emission nebula while the other recedes from it. This scenario was first advocated by Schmid (1996), who performed Monte Carlo simulations to investigate basic properties of Raman scattered $\mathrm{O}$ vi features. In his model, the red asymmetry aries when the receding region of neutral hydrogen is larger in extent than the approaching region.

Another possibility is that some part of the emission nebula approaches the neutral scattering region whereas the remaining part recedes from the $\mathrm{H}$ I region. In this picture, the emission region is identified with the accretion flow around the white dwarf. Based on this accretion flow model, Lee \& Kang (2007) successfully fitted the profile of the Raman O vi 6825 feature. In the current work, in order to investigate further the accretion flow model, we turn our attention to the weaker Raman 7082 feature.

Even though O vi $\lambda \lambda$ 1032, 1038 are formed in the same region, their flux ratio is observed to vary from 2 to 1 . If the emission nebula is sufficiently optically thin, then their flux ratio will be exactly 2 , because the statistical weight for the $j=3 / 2 \rightarrow j=1 / 2$ transition is twice that for the $j=1 / 2 \rightarrow j=1 / 2$ transition. This ratio will approach 1 in the infinite optical depth limit where the thermal spectrum is attained (e.g., Kang \& Lee 2008). Various flux ratios of resonance doublets N v $\lambda \lambda 1239,1243$ and C IV $\lambda \lambda 1548,1551$ have been reported for the symbiotic star CI Cyg by Mikolajewska et al. (2006). A similar behavior in another resonance doublet C IV $\lambda \lambda$ 1548, 1551 was found by Feibelman (1983), who investigated the spectra of a dozen planetary nebulae obtained with the International Ultraviolet Explorer (IUE).

In this regard, an interesting phenomenon has been noted that the profile of the double-peaked Raman 7082 feature is systematically different from that of the Raman 6825 feature. That is, the blue peak is relatively more suppressed in the Raman 7082 feature than in the Raman 6825 feature. This kind of systematic profile difference was pointed out by Schmid et al. (1999), who attributed it to the $\mathrm{TiO}$ absorption bands. However, in this work we adopt the view that the flux ratio of O VI $\lambda 1032$ and O VI $\lambda 1038$ differs locally in the $\mathrm{O}$ VI emission region. In the accretion flow scenario we may explain this phenomenon by assuming that the flux ratio in the blue emission region is larger than in the red emission region. This in turn implies that the red emission region is more optically thick than the blue emission region.

If the emission region is effectively monochromatic and the Doppler factors are attributed to the bulk motion of the neutral scattering region, then we expect that the two Raman scattered O VI features exhibit almost the same profiles differing only in total fluxes. Therefore, a quantitative comparison of the line profiles will shed much light on the mass transfer processes in symbiotic stars.

In this paper, we present our quantitative compari-
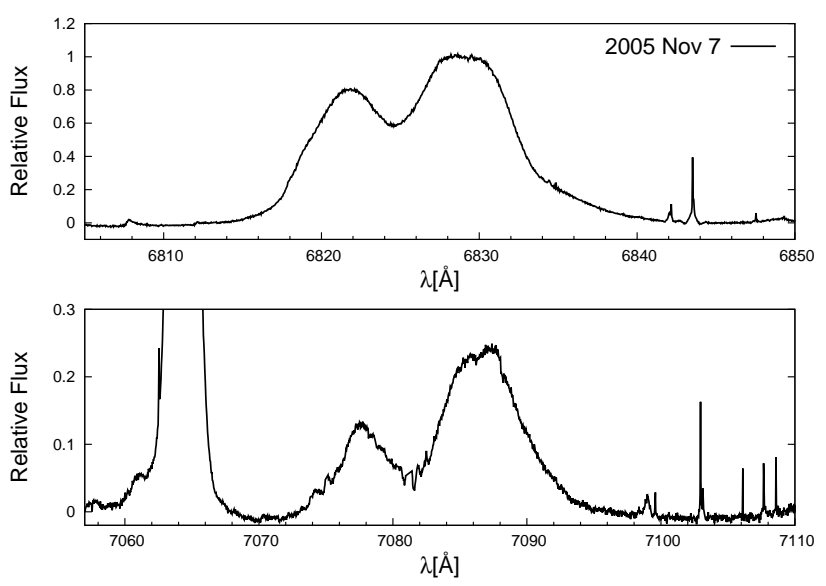

Figure 1. Parts of the BOES spectrum of the symbiotic star V1016 Cyg obtained in 2005. The upper panel shows the Raman 6825 feature and the lower panel shows the Raman 7082 feature. Note that the vertical scale of the lower panel is reduced by a factor of 4 compared to that of the upper panel in order to make the red peak of the Raman 7082 feature appear similar to that of the Raman 6825 feature.

son analysis of the two Raman scattered O vi profiles in the symbiotic nova V1016 Cyg. Adopting the accretion flow model, we construct emissivity maps and $\mathrm{O}$ VI optical depth distribution in the accretion flow around the white dwarf.

\section{ObServation}

\subsection{High Resolution Spectroscopy of V1016 Cyg}

V1016 Cyg is a D-type symbiotic nova that erupted with a magnitude change $\Delta m \sim 5-7 \mathrm{mag}$ in 1964 , which is thought to occur from the thermonuclear runaway process on the surface of the white dwarf component as it accreted material from the Mira component (e.g., McCusky 1965; Mikolajewska \& Kenyon 1992). Little is known about the fundamental parameters of V1016 Cyg including the mass, distance and orbital period (e.g., Whitelock 1987). The distance to V1016 Cyg is quite uncertain with the estimates ranging from less than $1 \mathrm{kpc}$ to $10 \mathrm{kpc}$ (e.g., Ivision et al. 1991; Lorenzetti et al. 1985; Watson et al. 2000). Brocksopp et al. (2002) presented their Hubble Space Telescope (HST) image of V1016 Cyg to propose that the projected binary separation of $84 \pm 2$ AU assuming the distance of 2 kpc. Schmid \& Schild (2002) suggested an orbital period longer than a century from their monitoring of the position angle of the linear polarization of the Raman O VI 6825 feature. A photometric variation with a period of 15 years was proposed by Parimucha et al. (2003), who attributed the activity to the orbital motion.

We obtained a high resolution spectrum of V1016 Cyg on the night of 2005 November 7 using the Bohyunsan Optical Echelle Spectrograph (BOES), which is an optical fiber fed system installed on the $1.8 \mathrm{~m}$ telescope at the Bohyunsan Optical Observatory. The spectral coverage of the BOES is from $3800 \AA$ to 
$10500 \AA$, which can be obtained with one single exposure. The optical fiber with a diameter of $300 \mu \mathrm{m}$ was used to yield a spectroscopic resolution $\sim 30,000$ and the exposure time was 7000 seconds. Use was made of the 'IRAF' and a standard procedure was followed to reduce the data. Figure 1 shows two parts of the spectrum that contain Raman scattered O vi features, where the 6825 feature is shown in the upper panel and the 7082 feature is found in the lower panel.

In particular, Lee \& Kang (2007) used the part of the spectrum shown in the upper panel for their profile fitting analysis. The 7082 feature is much weaker than the 6825 feature, which is attributed to the smaller scattering cross section for O VI $\lambda 1038$ than O VI $\lambda 1032$ by a factor $\sim 5$ (e.g., Allen 1980; Nussbaumer et al. 1989; Schmid 1989). In this work, we focus on the differing line profiles of these two Raman features. It is quite noticeable that the 7082 feature exhibits relatively more suppressed blue peak than the 6825 feature does.

\subsection{Profile Analysis}

When a far ultraviolet photon with frequency $\nu_{i}$ more energetic than Ly $\alpha$ is incident on a hydrogen atom in the ground state and subsequently scattered leaving the hydrogen atom in the $2 s$ state, then the frequency $\nu_{o}$ of the scattered photon is determined by the relation of energy conservation

$$
\nu_{o}=\nu_{i}-\nu_{\mathrm{Ly} \alpha}
$$

where $\nu_{\mathrm{Ly} \alpha}$ is the frequency of $\mathrm{Ly} \alpha$.

The adopted values of line center wavelengths of the far UV O VI resonance doublet are

$$
\lambda_{1}=1031.912 \AA, \quad \lambda_{2}=1037.613 \AA,
$$

respectively, with the $\operatorname{Ly} \alpha$ line center wavelength of $\lambda_{\mathrm{Ly} \alpha}=1215.670 \AA$.

The broad profiles that characterize Raman scattered features are also a direct consequence of energy conservation. This can be seen by observing the relation

$$
\frac{\Delta \nu_{o}}{\nu_{o}}=\frac{\Delta \nu_{i}}{\nu_{o}}=\left(\frac{\nu_{i}}{\nu_{o}}\right) \frac{\Delta \nu_{i}}{\nu_{i}},
$$

which leads to a broader profile by a factor $\nu_{i} / \nu_{o}$. In the case of Raman O vI, this factor amounts to around 7, leading to significantly broadened profiles. The profile broadening results in another important property that the profile of a Raman scattered feature is determined mainly by the relative motion between the scatterer and the emitter and mostly independent of the observer's line of sight.

To be more specific, we let $\mathbf{v}_{s}$ and $\mathbf{v}_{e}$ be the velocities of the scatterer and the emitter in the observers rest frame, respectively. We also let $\hat{\mathbf{k}}_{i}$ and $\hat{\mathbf{k}}_{f}$ be the unit wavevector of the incident $\mathrm{O}$ vi line photon with wavelength $\lambda_{i}$ and the Raman scattered optical photon with wavelength $\lambda_{o}$, respectively. Then the observed wavelength $\lambda_{\text {obs }}$ is

$$
\frac{\Delta \lambda_{o}}{\lambda_{o}}=\frac{\lambda_{o}}{\lambda_{i}} \frac{\hat{\mathbf{k}}_{i} \cdot\left(\mathbf{v}_{s}-\mathbf{v}_{e}\right)}{c}-\frac{\hat{\mathbf{k}}_{f} \cdot \mathbf{v}_{s}}{c}
$$
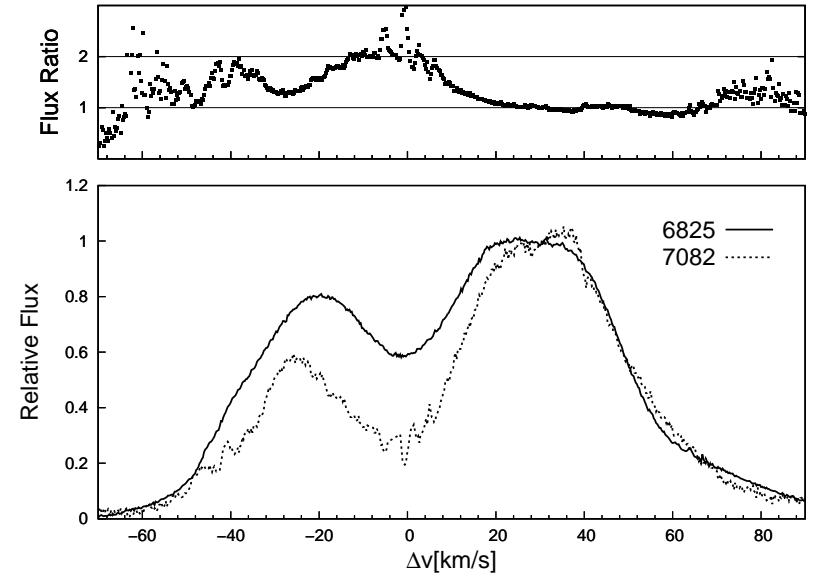

Figure 2. Profile comparison of the two Raman scattered features. The horizontal axis is the Doppler factor computed from the atomic line center. The upper panel shows the flux ratio of Raman scattered O vi $\lambda 1032$ at $6825 \AA$ and Raman scattered O vi $\lambda 1038$ at $7082 \AA$. In the lower panel, the solid line shows the Raman 6825 and the dotted line is for the Raman 7082 feature. The normalization is made in such a way that the red peaks are coincident.

where $\lambda_{\mathrm{obs}}=\Delta \lambda_{o}+\lambda_{o}$ is the observed wavelength (e.g., Nussbaumer et al. 1989; Schmid 1989). Because the factor $\lambda_{o} / \lambda_{i}$ is around 7 and $\mathbf{v}_{s}$ is small compared to $\mathbf{v}_{e}$, we neglect the last term in the above equation involving the observers line of sight and consider only the relative kinematics of the emission region with respect to the scattering region. In this sense, we are effectively viewing the emission region in the frame where the giant component is stationary.

We define the Doppler factor $\Delta V$, as the velocity component of the O VI ion with respect to the neutral scattering region. In Figure 2, we plot the two Raman scattered features on the Doppler factor axis in order to perform a quantitative profile comparison. The solid line shows the Raman 6825 feature and the dotted line is for the Raman 7082 feature. The following three steps have been followed to determine the Doppler factors for the two Raman scattered features.

Firstly, we shift the Raman features to the optical rest frame of V1016 Cyg determined by the 4 optical emission lines shown in Table 1 . We investigate the ratio of their observed wavelengths and the atomic line centers in order to obtain the average Doppler factor. The average value for those emission lines in Table 1 is determined to be 1.000122 , which is in turn multiplied to the two Raman scattered features to transform into the optical rest frame of V1016 Cyg.

Secondly, we multiply the refractive index of air to transform to the vacuum rest frame of V1016 Cyg. This is necessary because the spectral analysis of far UV O vi emission lines should be performed in the vacuum wavelength space. The adopted values for the air refractive indices are $n_{\text {air }}(6825)=1.00027597$ and $n_{\text {air }}(7082)=1.00027572 .^{1}$

\footnotetext{
${ }^{1}$ http://refractiveindex.info
} 
Table 1

Optical forbidden lines of V1016 Cyg to define the optical rest frame of V1016 Cyg.

\begin{tabular}{lccc}
\hline Line & $\begin{array}{c}\text { Atomic } \\
\text { Line Center } \\
\lambda_{c}(\AA)\end{array}$ & $\begin{array}{c}\text { Observed } \\
\text { Wavelength } \\
\lambda_{\text {obs }}(\AA)\end{array}$ & $\begin{array}{c}\text { Ratio } \\
\lambda_{c} / \lambda_{\text {obs }}\end{array}$ \\
\hline He I 7065 & 7065.195 & 7064.13 & 1.000151 \\
S II 6730 & 6730.815 & 6730.26 & 1.000083 \\
N II 6583 & 6583.450 & 6582.77 & 1.000103 \\
O I 6300 & 6300.304 & 6299.36 & 1.000150 \\
\hline Average & & & 1.000122 \\
\hline
\end{tabular}

Thirdly, we obtain the vacuum wavelength of the incident $\mathrm{O}$ vi line radiation using Equation (1).

In Figure 2, it is notable that the center dip appears at around $0 \mathrm{~km} \mathrm{~s}^{-1}$. Even though the double peak profiles are asymmetric, we may reasonably think that the central dip with the null Doppler factor divides the blue and red emission parts in the spectrum. This also implies that the neutral scattering region has no systematic bulk motion with respect to the rest frame of V1016 Cyg defined by the optical emission lines. In this figure, the red peaks of the two Raman features have been normalized so that their mean strengths are equal. We then plot the ratio of the flux in the two features as a function of the Doppler factor $\Delta V$ in the upper panel.

We immediately notice that the flux ratio at the central dip has the largest value of 2 , which is the theoretical maximum ratio of O VI $\lambda 1032$ to O VI $\lambda 1038$. The red and blue wing regions also exhibit the maximum flux ratio of 2 as in the central dip region. However, the weak flux of the Raman 7082 feature in the wing regions renders the flux ratio in these regions unreliable. We note that the flux ratio in the blue peak region is around 1.5, which is clearly distinct from the red peak region and the central dip part. With the normalization of equal red peak strengths the flux ratio is observed to range from 1 to 2 . Because this happens to be the full range that is theoretically allowed, we may conclude that this normalization is the unique possibility.

Figure 2 also shows that the emission region can be divided into three parts based on the flux ratio of approximately $1,1.5$ and 2 , which reflects the optical thickness of $\mathrm{O}$ VI doublet lines. The part of the emission region with the flux ratio 1 is mainly found around the red peak, where O VI emission is dominantly produced. The intermediate flux ratio of $\sim 1.5$ is seen around the blue peak, where O VI emission is less prominent than the red counterpart. The emission region with flux ratio near 2 corresponds to a highly extended outer region moving slowly or the region moving across the line connecting the two stars. It appears that the flux ratio is anticorrelated with the flux intensity so that the flux ratio approaches 2 as the flux decreases.

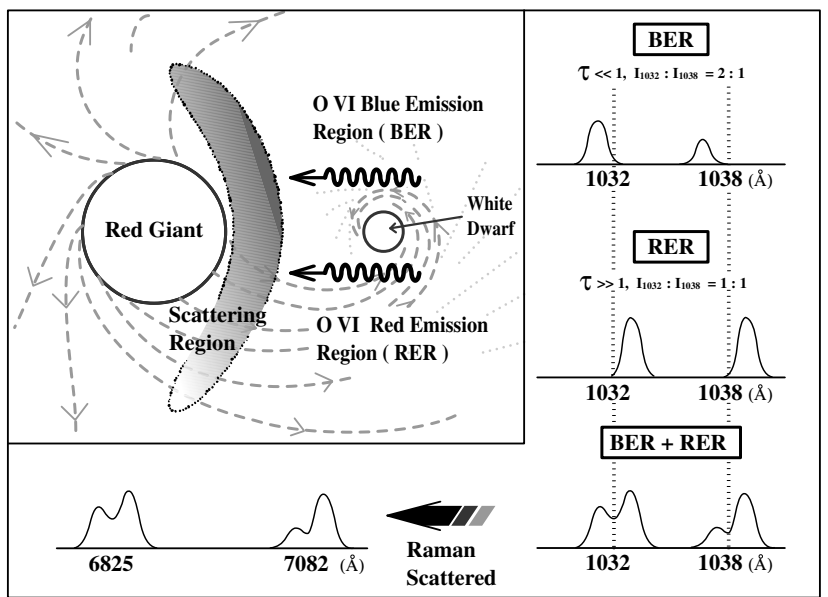

Figure 3. A schematic illustration (not to the scale) of the mass transfer and Raman scattering processes in the symbiotic star V1016 Cyg. The orbital motion is taken to be conterclockwise. Observer's line of sight is not specified because the line profiles of the Raman O VI features are determined by the relative kinematics of the emission region and the scattering region. The emission region is identified with the accretion flow around the hot white dwarf and is divided into the "blue emission region" and the "red emission region" denoted by BER and RER, respectively.

\section{ACCRETION FLOW AND EMissivity MAPPING}

\subsection{Accretion Emission Region}

Lee \& Park (1999) proposed that the double-peak profile of the Raman O vi feature should be attributed to the kinematics of the emission region. According to them, the $\mathrm{O}$ VI emission region is identified as an accretion flow around the white dwarf as it gravitationally captures some fraction of the slow stellar wind from the giant component (e.g., Livio et al. 1989; Mastrodemos \& Morris 1998; de Val-Borro et al. 2009). In this scenario, the red asymmetry is obtained due to the converging flow near the entrance region in the accretion flow, which may exhibit density enhancement with positive Doppler factors. The opposite side of the accretion flow tends to be divergent due to the opposing flow of the slow stellar wind from the giant, which may result in a low density region. In this picture, the accretion disk is assumed to be Keplerian and the peak separation in the double-peak profile corresponds to the velocity scale at the outer edge of the accretion flow.

In this work, we do not perform any hydrodynamic calculations but instead adopt a simple kinematic model for the scattering region and the emission region. We take the view that the emission region is characterized by a Keplerian disk with the inner and outer radii $R_{i}$ and $R_{o}$, respectively. With the mass of the white dwarf $M_{*}$, the Keplerian velocity at the inner radius is given by

$$
v_{i}=\sqrt{\frac{G M_{*}}{R_{i}}} .
$$

Correspondingly, we note that the velocity $v_{o}=$ $v_{i}\left(R_{i} / R_{o}\right)^{1 / 2}$ at the outer radius. Due to the paucit0y 
of information about the fundamental parameters of V1016 Cyg mentioned earlier, in this work for the sake of scaling, we set $M_{*}=1 \mathrm{M}_{\odot}$ so that the rotation speed $v=30 \mathrm{~km} \mathrm{~s}^{-1}$ at $r=1 \mathrm{AU}$.

In Figure 3, we show a schematic illustration adopted in this work for the line formation of the Raman 6825 and 7082 features in V1016 Cyg. Note that the figure is not to scale and that the orbital motion occurs counterclockwise. If we adopt the values presented in Brocksopp et al. (2002) the binary separation should be much larger than illustrated. As mentioned in the previous section, the line profile analysis is independent of the orbital parameters and the observers line of sight, which are omitted in the figure.

The accretion flow is roughly divided into the "blue emission region (BER)" and the "red emission region (RER)", where the BER is the part of the accretion flow approaching the neutral scattering region or $\Delta V<$ 0 . In the RER, the accretion flow recedes from the scattering region, or equivalently $\Delta V>0$.

It is expected that the RER is denser than the BER, because the accretion flow in the BER is opposed to the slow stellar wind from the giant. The flux ratio of O VI $\lambda 1032$ to O VI $\lambda 1038$ is expected to be smaller in the RER than that in the BER, which leads to the relatively more suppressed blue peak of the Raman 7082 feature than the Raman 6825 counterpart.

\subsection{Emissivity Maps}

Doppler tomography is a popular technique to study detailed structures of the accretion disk in a cataclysmic variable (e.g., Marsh \& Horne 1988). A series of high resolution spectra covering the entire orbital phase are obtained and reorganized to map the emission region according to the emission profile. The emission from a specific structure such as the hot spot has a varying Doppler factor as a function of the orbital phase. By correcting the Doppler factor to a direction that is fixed in the binary system, we can make a map which indicates the region of the emission in the two-dimensional velocity space. Therefore the Doppler tomography is particularly useful to locate the hot spot in the accretion disk in a cataclysmic binary system.

However, the same technique is an inappropriate option in the case of V1016 Cyg with an orbital period exceeding several decades and a long period variable companion. We produce the emissivity maps of the accretion flow based on the profiles of Raman scattered $\mathrm{O}$ VI features in the real 2-dimensional space instead of the 2-dimensional velocity space. An emissivity map in this work is basically meant to be a plot of the weight assigned to a region characterized by a given Doppler factor, where the weight is simply proportional to the Raman flux at the Doppler factor. Figure 4 shows the emissivity map obtained from the observed Raman 6825 feature. The white dwarf lies at the center of the figure and the distance unit is $1 \mathrm{AU}$.

We briefly describe how the map is obtained using the observed Raman 6825 profile. A coordinate system is set up so that the origin coincides with the location

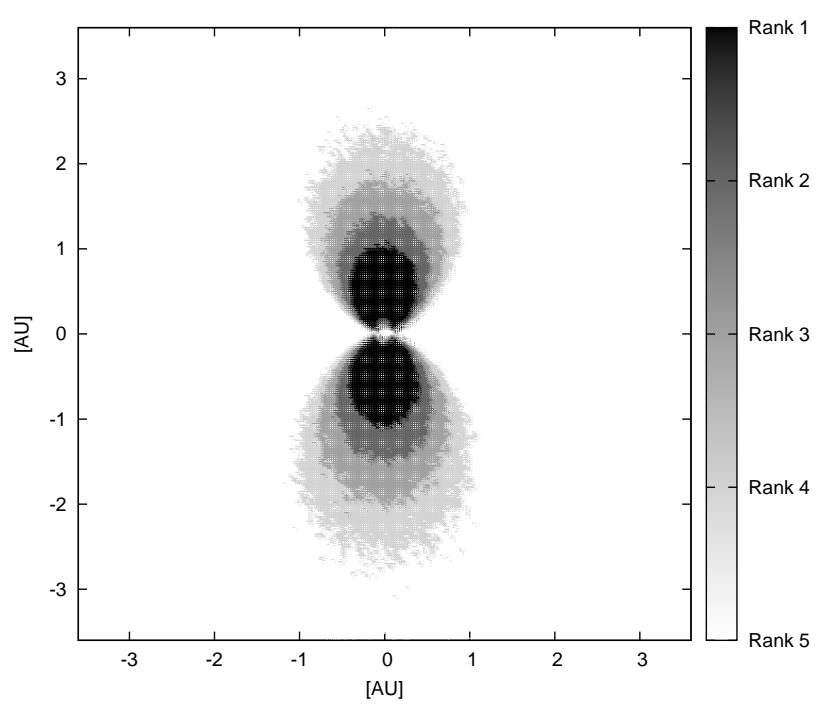

Figure 4. Emissivity map for the Raman 6825 feature of V1016 Cyg. At the center lies the white dwarf and the unit of distance along the axes is $1 \mathrm{AU}$. To each region with a given Doppler factor a weight proportional to the Raman flux is assigned, which is shown by a grey scale. The weights are grouped into 5 rankings. The subregion with ranking 1 has the overall twice emissivity than that with ranking 2, which in turn has twice emissivity than the subregion with ranking 3, and so on. The red asymmetric Raman profile is responsible for the more extended coverage of the RER than the BER.

of the white dwarf and the neutral scattering region is placed very far away in the $-x$ direction from the white dwarf, as illustrated in Figure 3. In this coordinate system, the region with negative $y$-coordinate values corresponds to the RER. We use a Monte Carlo technique to construct the back projection from the observed profile to the region of the accretion flow.

The profile of the Raman 6825 feature is normalized so that the maximum value of the red peak is 1 against the horizontal axis in Figure 2 representing the Doppler factor $\Delta V$ with the interval of $\delta V=0.3 \mathrm{~km} \mathrm{~s}^{-1}$. In this manner we obtain a profile function $\Phi_{1}(\Delta V)$ for the Raman 6825 feature as a function of the Doppler factor $\Delta V$. Here, $\Delta V$ ranges from $-80 \mathrm{~km} \mathrm{~s}^{-1}$ to $+80 \mathrm{~km} \mathrm{~s}^{-1}$. In addition, we prepare an array $\Psi(\Delta V)$, which is to be compared to $\Phi(\Delta V)$. Here, we assume that the Raman conversion efficiency is constant for all O VI $\lambda 1032$ photons irrespective of the Doppler factor.

Two uniform random numbers $u_{1}, u_{2}$ are generated to pick a position $(r, \theta)$ in an accretion disk with the inner radius $R_{i}$ and the outer radius $R_{o}$. With the prescription

$$
r=\sqrt{R_{i}^{2}+u_{1}\left(R_{o}^{2}-R_{i}^{2}\right)}, \quad \theta=2 \pi u_{2},
$$

where $u_{1}$ and $u_{2}$ are uniform random deviates between 0 and 1 , the selection of a region in the disk is made in a uniform way. We determine the Doppler factor $\Delta V$ of this chosen region assuming that the accretion flow is purely Keplerian. Then we add a numerical 


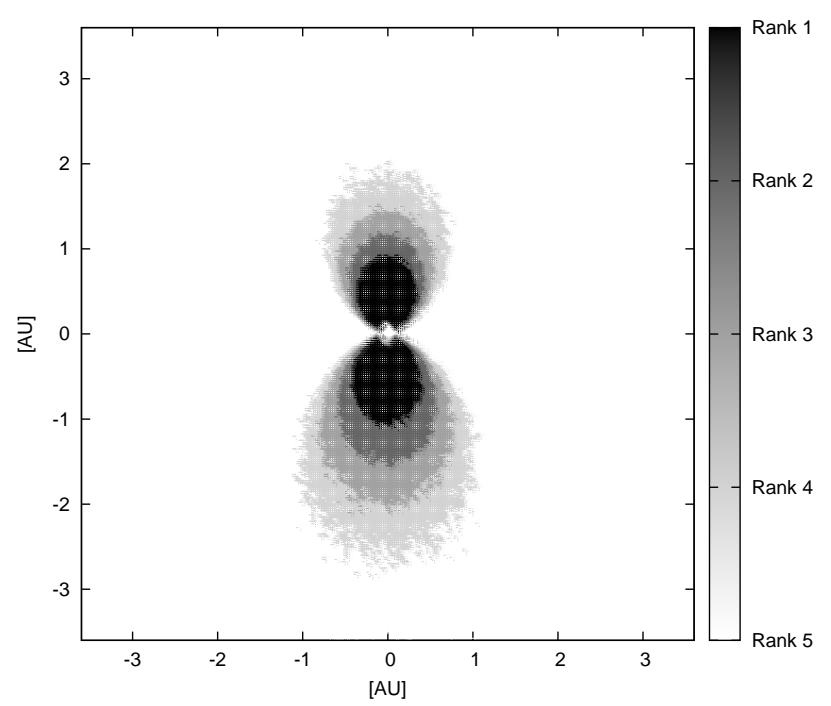

Figure 5. Emissivity map for the Raman 7082 feature of V1016 Cyg. The physical scale and the configuration are the same as in Figure 4. The same ranking system as in Figure 4 is applied. The shaded blue emission region is considerably smaller than the counterpart for the Raman 6825 feature.

value of $10^{-3}$ to the test profile function $\Psi(\Delta V)$ if $\Psi(\Delta V) \leq \Phi(\Delta V)$. Otherwise we discard this selection. We iterate this procedure until $\Psi(\Delta V)=\Phi(\Delta V)$ for all possible values of $\Delta V$. Thus, the number of points in the region defined by a given Doppler factor will be proportional to the Raman flux at this Doppler factor. The collection of selected points constitutes an emissivity map for the Raman scattered O VI $\lambda$ 1032, which is shown in Figure 4.

The contour having a fixed Doppler factor takes a characteristic elongated shape that is symmetric with respect to the central vertical axis. This leads to emissivity maps symmetric about the central vertical axis. We divide the emission region into 5 subregions by assigning a rank based on the O vi flux density.

The subregion with rank 1 is determined so as to cover the inner accretion flow region with Keplerian velocity $\sim 35 \mathrm{~km} \mathrm{~s}^{-1}$. We plot the subregion with rank 2 so that it has overall half the emissivity of that with rank 1. In turn, the subregion with rank 3 has half the emissivity of that with rank 2 , and so on. As $r$ increases, the flux density decreases as is expected from the observed data. We clearly see that the RER is covered more extensively, which corresponds to the red asymmetric double-peak profile of the Raman 6825 feature.

A similar procedure is also applied to produce the emissivity maps of the Raman 7082 feature, for which the result is shown in Figure 5. The same ranking system as in Figure 4 is applied to this plot. In this case, the RER is much more prominent than the BER compared to the case of Figure 4. The coverage of the RER in Figure 5 is almost similar to the counterpart of Figure 4, whereas the BER coverage for the Raman 7082 feature is much smaller than that of the Raman 6825

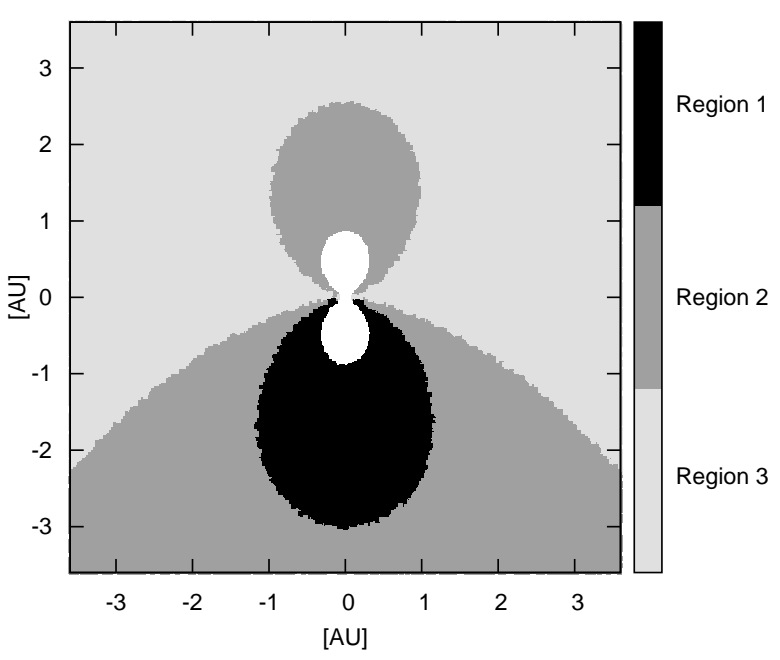

Figure 6. Map of the flux ratio of the OVI $\lambda \lambda 1032$ and 1038. The physical scale and the configuration are the same as in Figures 4 and 5. We divide the emission region into 3 subregions according to the flux ratio. The three regions are shown with the grey scale, where Region 1 (black) has the lowest flux ratio. Region 2 (dark grey) exhibits the flux ratio between 1.15 and 1.6. Region 3 (light grey) with the ratio around 2:1 is quite extended with very small Doppler factors.

feature. The similar coverage of the RER between the two Raman features is attributed to the unique normalization of equal red peak strengths as discussed in the previous section. With this unique normalization, the conspicuously smaller coverage of the BER for the Raman 7082 feature is another way of describing the disparity of the two Raman O vi profiles.

\subsection{Flux Ratio Map}

As mentioned above, we attribute the disparity of the two Raman O vi profiles to the local variation of the doublet O vi flux ratio $F(1032) / F(1038)$ in the O vi emission region. Referring to Figure 2, we limit our attention to the range of the Doppler factor space from $-30 \mathrm{~km} \mathrm{~s}^{-1}$ to $+30 \mathrm{~km} \mathrm{~s}^{-1}$, where most $\mathrm{O}$ vi emission is found.

Noting that the flux ratio of O vi $\lambda 1032$ and O vi $\lambda 1038$ varies from 1 to 2 , we divide this full interval $I=[1,2]$ into three subintervals $I_{1}, I_{2}$, and $I_{3}$ where $I_{1}=[1,1.15), I_{2}=[1.15,1.6)$ and $I_{3}=[1.6,2]$. The data points near the central dip showing the flux ratio exceeding the theoretically allowed maximum of 2 are included in the subinterval $I_{3}$. We then back-project to the space of the accretion flow around the white dwarf. This allows us to divide the accretion flow into three regions (Region 1, Region 2 and Region 3 ) corresponding to the three intervals $I_{1}, I_{2}$ and $I_{3}$.

We expect that the region with flux ratio near 1 is optically very thick, which is plausibly found in the inner region in the accretion flow. Similarly, the outer region with the lowest density may be characterized by the flux ratio reaching the highest possible value of 2 . 
In Figure 6, we present the three regions Region 1, Region 2 and Region 3 around the white dwarf component using grey scale, where the physical scale and the configuration are the same as in Figures 4 and 5. The white region found near the white dwarf results from the exclusion of the data points having the Doppler factors outside of $\left[-30 \mathrm{~km} \mathrm{~s}^{-1},+30 \mathrm{~km} \mathrm{~s}^{-1}\right]$.

As is shown clearly in the figure, Region 1 with the lowest flux ratio and hence regarded as being of the highest density is found in the inner part of the RER. It is interesting to note that Region 1 does not intersect with the BER. As is shown in Figure 2, Region 1 consists of those regions with the Doppler factor between $+15 \mathrm{~km} \mathrm{~s}^{-1}$ and $+30 \mathrm{~km} \mathrm{~s}^{-1}$. Region 3 corresponds to the outer region with weak emission.

Region 2 shaded by dark grey is found mostly in the outer region of Region 1 and is also found in the inner region of the BER. As is illustrated in Figure 2, Region 2 is found mainly in the region with the Doppler factor near $-25 \mathrm{~km} \mathrm{~s}^{-1}$ and in the region with the Doppler factor $\sim+15 \mathrm{~km} \mathrm{~s}^{-1}$.

\section{Summary AND Discussion}

In this work, we have performed a quantitative comparison of the line profiles of the two Raman scattered features at $6825 \AA$ and $7082 \AA$ from the high resolution spectrum of the symbiotic star V1016 Cyg obtained with the BOES in 2005. We attribute the disparity in the profiles to the local variation of the flux ratio of O vi $\lambda 1032$ and OVI $\lambda 1038$ in the O vi emission region that is regarded as the accretion flow around the white dwarf. The profile comparison is made by transforming the two observed optical features first to the vacuum optical rest frame and then to the vacuum UV rest frame of O Vi $\lambda \lambda 1032$ and 1038, where the Doppler factors with respect to the neutral scattering region are determined. With the constraint that the flux ratio of O VI $\lambda 1032$ and O VI $\lambda 1038$ varies between 1 and 2, a unique normalization is obtained where the red peaks of the two Raman features coincide in the Doppler factor space as shown in Figure 2. We provide the emissivity maps that assign to each region with a specified Doppler factor a weight that is proportional to the Raman flux. These maps are another way of presenting the red asymmetry of the Raman profiles. A map of the flux ratio is also produced in order to infer the matter distribution in the accretion flow. The inner part of the red emission region in the accretion flow shows the flux ratio of $\sim 1$, which implies that O VI emission is optically very thick. The region with the lowest optical depth is quite extended to the outer region with negligible Doppler factors.

It appears that other Raman features of He II and Ne VII exhibit broad single peak profile even though they are usually very weak (e.g., Lee 2012; Lee, Heo \& Lee 2014; Birriel 2004). If this is the case, it is unclear why the profiles of these Raman features differ systematically from those of Raman $\mathrm{O}$ vi features. The relative weakness of Raman He II and Ne VII features compared with Raman O vi features strongly implies that HeII and Ne VII emission regions are much less dense than $\mathrm{O}$ VI emission region in the accretion flow. This is further supported because He II and Ne VII have larger scattering cross section than $\mathrm{O}$ VI resonance(e.g., van Groningen 1993; Nussbaumer et al. 1989; Lee et al. 2014) emissivity scaling with the density squared, we may expect that He II and Ne VII emission are produced in a small region of exceptionally enhanced density having a very narrow range of Doppler factors. In this case the resulting Raman profile will be single-peaked with the peak located at the Doppler factor of the He II and Ne VII emission spot.

Spectropolarimetry of Raman O VI 6825 and 7082 provided by Harries \& Howarth (1997) shows that the extreme red wing part of the Raman O VI 6825 and 7082 features is polarized with the position angle that differs by 90 degrees from that of the main part of the features (see also Schild \& Schmid 1996). This is very interesting in view of the bipolar morphology of the emission nebula (Solf 1983; Brocksopp et al. 2002; Lee \& Park 1999). In our model, the polarization flip in the extreme red part implies the existence of an additional neutral scattering region that recedes from the $\mathrm{O}$ vi emission region in the direction normal to the orbital plane. In this case the additional scattering region may be neutral clumps that may have formed through Rayleigh-Taylor instability in the bipolar regions. Another possibility is that instead of assuming an additional scattering region we can place an additional emission region that recedes from the giant in the direction perpendicular to the orbital plane. This emission flow may be identified with the collimated bipolar outflow.

Due to the weakness of the Raman 7082 feature compared to the Raman 6825 feature, it requires a good amount of telescope time to secure spectra with high signal to noise ratio and sufficient spectral resolution. However, considering the disparity in the profiles can be an important probe of the accretion flow around the white dwarf, we expect that high quality spectroscopic data in other symbiotic systems will reveal much information on the mass transfer processes in symbiotic stars.

\section{ACKNOWLEDGMENTS}

We are grateful to the anonymous referee for very useful comments that improved the presentation of the paper. We thank the staff of the Bohyunsan Optical Observatory for their help in obtaining the high resolution spectra of V1016 Cyg. This research was supported by the Basic Science Research Program through the National Research Foundation (NRF-2014R1A1A2054887).

\section{REFERENCES}

Allen, D. A. 1980, Candidate Symbiotic Stars in the Large Magellanic Cloud, ApJ, 20, 131

Angeloni, R., Contini, M., Ciroi, S., \& Rafanelli, P. 2010, The Spectral Energy Distribution of D-Type Symbiotic Stars: the Role of Dust Shell, MNRAS, 402, 2075

Bach, K., \& Lee, H.-W. 2014, The Kramers-Heisenberg Formula and the Gunn-Peterson Trough, JKAS, 47, 187 
Birriel, J. 2004, Raman-Scattered He II at $4851 \AA$ in the Symbiotic Stars HM Sagittae and V1016 Cygni, ApJ, 612, 1136

Brocksopp, C., Bode, M. F., Eyres, S. P. S., Crocker, M. M., Davis, R., \& Taylor, A. R. 2002, The Central Binary and Surrounding Nebular of the Symbiotic Star V1016 Cygni, ApJ, 571, 947

de Val-Borro, M., Karovska, M., \& Sasselov, D. 2009, Numerical Simulations of Wind Accretion in Symbiotic Binaries, ApJ, 700, 1148

Feibelman, W. 1983, Profiles and Intensity Ratios of the C IV $\lambda$ 1548, 1550 Emission Lines in Planetary Nebulae, A\&A, 122,335

Harries, T. J., \& Howarth, I. D. 1997, Raman Scattering in Symbiotic Stars. II. Numerical Models, A\&AS, 121, 15

Iben, I. Jr., \& Tutukov, A. V. 1984, Supernovae of Type I as End Products of the Evolution of Binaries with Components of Moderate Initial Mass (M not Greater than about 9 Solar Masses), ApJS, 54, 335

Ivison, R. J., Bode, M. F., Roberts, J. A., Meaburn, J., Davis, R. J., Nelson, R. F., \& Spencer, R. E. 1991, A Multi-Frequency Study of Symbiotic Stars. I - Nearsimultaneous Optical and Radio Observations, MNRAS, 249,374

Kang, E.-H., \& Lee, H.-W. 2008, Effects of Collisional DeExcitation on the Resonance Doublet Flux Ratios in Symbiotic Stars and Planetary Nebulae, JKAS, 41, 49

Lee, H.-W. 2012, Raman Scattered He II 4332 in the Symbiotic Star V1016 Cygni, ApJ, 750, 127

Lee, H.-W., \& Kang, S. 2007, Raman-scattered O VI 6825 and the Accretion Disk Emission Model in the Symbiotic Stars V1016 Cygni and HM Sagittae, ApJ, 669, 1156

Lee, H.-W., \& Park, M.-G. 1999, Toward the Evidence of the Accretion Disk Emission in the Symbiotic Star RR Telescopii, ApJ, 515, L89

Lee, H.-W., Heo, J.-E., \& Lee, B.-C. 2014, Raman-Scattered Ne VII 973 at 4881 in the Symbiotic Star V1016 Cygni, MNRAS, 442, 1956

Livio, M., Prialnik, D., \& Regev, O. 1989, Accretion onto Hot White Dwarfs in Relation to Symbiotic Novae, ApJ, 341,299

Lorenzetti, D., Saraceno, P., \& Strafella, F. 1985, On the IR Variability of Symbiotic Stars - The Case of V1016 Cygni, HM Sagittae and V1329 Cygni, ApJ, 298, 350

Mastrodemos, N., \& Morris, M. 1998, Bipolar Preplanetary
Nebulae: Hydrodynamics of Dusty Winds in Binary Systems. I. Formation of Accretion Disks, ApJ, 497, 303

Marsh, T. R., \& Horne, K. 1988, Images of Accretion Discs. II - Doppler Tomography, MNRAS, 235, 269

Mikolajewska, M. 2012, Symbiotic Stars: Observations Confront Theory, Baltic Astron., 21, 5

Mikolajewska, M., Friedjung, M., \& Quiroga, C. 2006, Line Formation Regions of the UV Spectrum of CI Cygni, A\&A, 460, 191

Mikolajewska, M., \& Kenyon, S. 1992, On the Nova-Like Eruptions of Symbiotic Binaries, MNRAS, 256, 177

McCuskey, S. 1965, Activity in H Emission Object, IAU Circ., 1916, 0

Nussbaumer, H., Schmid, H. M., \& Vogel, M. 1989, Raman Scattering as a Diagnostic Possibility in Astrophysics, A\&A, 211, L27

Parimucha, S., Chochol, D., Pribulla, T., Buson, L. M., \& Vittone, A. A. 2003, Fifteen-Year Period of Activity in the Symbiotic Nova V1016 Cyg, ASPC, 303, 80

Perlmutter, S., et al. 1998, Discovery of a Supernova Explosion at Half the Age of the Universe, Nature, 391, 51

Schild, H., \& Schmid, H. M. 1996, Spectropolarimetry of Symbiotic Stars. On the Binary Orbit and the Geometric Structure of V1016 Cygni, A\&A, 310, 211

Schmid, H. M. 1989, Identification of the Emission Bands at $6830,7088 \AA$ A\&A, 211, L31

Schmid, H. M. 1996, Simulations of the Raman-Scattered OVI Emission Lines in Symbiotic Stars, MNRAS, 282, 511

Schmid, H. M., \& Schild, H. 2002, Orbital Motion in Symbiotic Mira Systems, A\&A, 395, 117

Schmid, H. M., et al. 1999, ORFEUS Spectroscopy of the O BT VI Lines in Symbiotic Stars and the Raman Scattering Process, A\&A, 348, 950

Solf, J. 1983, Optical Confirmation of a Very Compact Bipolar Nebula Associated with the Symbiotic Star V1016 Cygni, ApJ, 266, L113

van Groningen, E. 1993, Further Evidence for Raman Scattering in Rr-Telescopii, MNRAS, 264, 975

Warner, B. 1995, Cataclysmic Variable Stars (Cambridge: Cambridge University Press)

Watson, S. K., Eyres, S. P. S., Davis, R. J., Bode, M. F., Richards, A. M. S., \& Kenny, H. T. 2000, Colliding Winds in V1016 Cygni, MNRAS, 311, 449

Whitelock, P. 1987, Symbiotic Miras, PASP, 99, 573 